\title{
A randomized, placebo-controlled, phase 1 study to evaluate the effects of TAK-063 on ketamine-induced changes in fMRI BOLD signal in healthy subjects
}

\author{
Deborah A. Yurgelun-Todd ${ }^{1}$ (D) $\cdot$ Perry F. Renshaw ${ }^{1} \cdot$ Paul Goldsmith $^{2} \cdot$ Tolga Uz $^{3} \cdot$ Thomas A. Macek $^{3}$
}

Received: 15 March 2019 / Accepted: 22 September 2019 / Published online: 26 November 2019

(C) The Author(s) 2019

\begin{abstract}
Rationale Phosphodiesterase 10A inhibitor TAK-063 has shown effects that suggest efficacy in schizophrenia treatment.

Objective This randomized, double-blind, placebo-controlled, incomplete-crossover study investigated effects of single oral administration of TAK-063 on ketamine-induced changes in blood oxygen level-dependent (BOLD) signal in healthy males.

Methods Healthy men aged 18 to 45 years with normal magnetic resonance imaging (MRI) scans and electroencephalogram measurements at screening were eligible. Each subject was randomized to one of nine treatment schedules: all subjects received placebo and two of three doses of TAK-063 followed by ketamine. The primary endpoint was ketamine-induced brain activity in select regions of the brain during resting state. Secondary endpoints included pharmacokinetic parameters of TAK-063, proportion of subjects with treatment-emergent adverse events (AEs), and percentage of subjects meeting criteria for abnormal safety laboratory tests and vital sign measurements.

Results The study comprised 27 subjects. Prior to ketamine infusion, TAK-063 exerted region-specific effects on resting state functional MRI (fMRI) BOLD signal. After ketamine administration, TAK-063 reduced the Cohen's effect size for resting-state fMRI BOLD signal in key brain regions examined, and exerted similar effects on BOLD signal during the working memory task across all doses. TAK-063 was safe and well tolerated.

Conclusions Our results are consistent with non-clinical studies of ketamine and TAK-063 and clinical studies of ketamine and risperidone. It is unknown whether these data are predictive of potential antipsychotic efficacy, and further analyses are required.
\end{abstract}

Keywords fMRI $\cdot$ BOLD $\cdot$ Ketamine $\cdot$ TAK-063 $\cdot$ PDE-10 inhibitor

\section{Introduction}

Schizophrenia is a complex disorder consisting of positive symptoms (paranoia, hallucinations, suspiciousness, and

Electronic supplementary material The online version of this article (https://doi.org/10.1007/s00213-019-05366-1) contains supplementary material, which is available to authorized users.

Deborah A. Yurgelun-Todd

Deborah.Yurgelun-Todd@hsc.utah.edu

1 The Brain Institute, University of Utah, 383 Colorow Drive, Salt Lake City, UT 84108, USA

2 Takeda Development Center Europe, Ltd., 61 Aldwych, London WC2B 4AE, UK

3 Takeda Development Center Americas, Inc., One Takeda Parkway, Deerfield, IL 60015, USA unusual thought content), negative symptoms (blunted affect, emotional withdrawal, and poor rapport), and cognitive impairments (attentional and memory deficits, impairments in executive function, and others) (Citrome 2014). Schizophrenia imposes a significant financial and humanistic burden because of factors such as institutionalization and adverse side effects from chronic medication use (Millier et al. 2014). Current antipsychotics have shown efficacy in treating positive symptoms associated with schizophrenia, but have had limited success with treating negative and cognitive symptoms (Citrome 2014). Thus, new treatment options with improved safety and tolerability profiles are needed to address these poorly controlled domains within schizophrenia (Citrome 2014).

Ketamine, an N-methyl-D-aspartate (NMDA) receptor antagonist, is used clinically as a dissociative anesthetic (Yamakura et al. 2000). Ketamine also increases psychotic symptoms in individuals with schizophrenia (Lahti et al. 2001). Sub-anesthetic doses of ketamine induce effects that 
resemble schizophrenia symptoms in healthy individuals, and have therefore been used as a model for schizophrenia in humans (Abi-Saab et al. 1998; Lahti et al. 2001) (reviewed in Frohlich and Van Horn 2014). Specifically, ketamine impairs working memory and psychomotor function in healthy volunteers (Driesen et al. 2013; Lofwall et al. 2006; Morgan et al. 2004) and induces changes in blood oxygen leveldependent (BOLD) signal as measured by functional magnetic resonance imaging (fMRI) (De Simoni et al. 2013; Driesen et al. 2013). In previous studies, ketamine-induced changes in BOLD signal have been shown to be reversed by risperidone, a dopamine receptor antagonist and atypical antipsychotic (Doyle et al. 2013; Shcherbinin et al. 2015).

TAK-063 is a selective inhibitor of phosphodiesterase 10A (PDE10A), an intracellular enzyme selectively expressed in medium spiny neurons of the striatum (Seeger et al. 2003; Suzuki et al. 2015). In preclinical studies in combination with NMDA antagonists, TAK-063 has been shown to have potential antipsychotic effects in animal models of schizophrenia (Suzuki et al. 2016). For example, TAK-063 reversed MK801-induced effects on hyperactivity in rats (Suzuki et al. 2016), as well as MK-801 and phencyclidine-induced effects on spatial working memory, attentional set-shifting, and executive function in rodent models of cognitive impairment (Shiraishi et al. 2016). TAK-063 also increased percent BOLD signal change during the resting state in rat caudate putamen and substantia nigra, and reduced ketamineinduced increases in percent BOLD signal change in cortical regions (hippocampus, cingulate [frontal] and retrosplenial [parietal] cortex) in anesthetized rats (Tomimatsu et al. 2016), suggesting that TAK-063 may alleviate physiological features associated with schizophrenia.

In clinical studies, TAK-063 has been shown to be safe and well tolerated (Goldsmith et al. 2017; Tsai et al. 2016). In addition, TAK-063 has shown effects in patients with schizophrenia that are suggestive of potential efficacy in the treatment of this disorder, including increases in electroencephalogram (EEG) gamma power, improvements in mismatch negativity, and some improvements in cognition (Macek et al. 2016a, b).

Although TAK-063 has shown signs of efficacy in preclinical models, its effects in the clinical setting have not been extensively characterized. In a study with a similar design to the one we report here, ketamine was shown to produce a broad increase in BOLD signal; co-administration of risperidone reduced the effect of ketamine on BOLD signal across most regions of interest (ROIs), including the medial prefrontal and cingulate regions and the thalamus (Doyle et al. 2013). Based on the pharmacological properties of TAK-063 and the potential antipsychotic activity observed in preclinical and clinical studies, it was predicted that TAK-063 would have similar effects on the ketamine-induced increase in BOLD signal. We hereby report the effects of TAK-063 on ketamine-induced brain changes in BOLD signal during the resting state and working memory tasks in healthy male subjects.

\section{Methods}

\section{Study design and subjects}

This was a randomized, placebo-controlled, investigator- and subject-blinded, three-period, incomplete crossover, singlecenter, phase 1 study that was conducted in the USA from June 27, 2013, to August 28, 2014. Data were collected at the University of Utah Neuropsychiatric Institute (Salt Lake City, UT), and the protocol was performed in compliance with Good Clinical Practice regulations and the Declaration of Helsinki. The Institutional Review Board's written approval of the protocol was received before commencement of this study. Informed consent was obtained from all individual participants included in the study. The study is registered as Effects of TAK-063 on Preventing Ketamine-Induced Brain Activity Changes as Well as Psychotic-Like Symptoms in Healthy Male Adults (Clinical Trials ID: NCT01892189; https://clinicaltrials.gov/ct2/show/NCT01892189).

Healthy adult men aged 18 to 45 were eligible for the study if they had a body mass index (BMI) between 18 and 32 $\mathrm{kg} / \mathrm{m}^{2}$, as well as normal magnetic resonance imaging (MRI) and EEG measurements at screening. Subjects were excluded if they had received any investigational compound or ketamine within 30 days and had an uncontrolled clinically significant neurologic, metabolic, or psychiatric disorder or other anomaly (including on MRI or EEG), which may have confounded study results. Subjects were also excluded if they had a history of drug or alcohol abuse or dependence, a positive result for illicit drugs or alcohol at screening or check-in, use of nicotine-containing products within 28 days before checkin, or a history of psychiatric disorders such as depression, bipolar disorder, and schizophrenia.

The Statistics Department at Takeda Development Center Americas, Inc., generated a randomization schedule before the start of the study, and this randomization schedule maintained the investigational drug blind. During randomization, subjects were assigned a four-digit randomization sequence that encoded assignment of subjects to the treatment schedule. Subjects were randomized to one of nine treatment schedules and received placebo + ketamine (regimen A) and two of the following three treatment regimens: $3 \mathrm{mg}$ of TAK-063 + ketamine (regimen B), $30 \mathrm{mg}$ of TAK-063 + ketamine (regimen C), and $300 \mathrm{mg}$ of TAK-063 + ketamine (regimen D). This design was updated to change the 300-mg TAK-063 dose to $10 \mathrm{mg}$ in regimen $\mathrm{D}$ following the observation of higher than expected rates of dystonia and nausea; the 300-mg dose was not included in the analysis. Treatment sequences are 
presented in Online Resource Supplementary Table S1. The study consisted of multiple visits with a different treatment protocol on each visit. The visits were standardized and included a screening period (day -28 to day -2 ), check-in (day -1 ), a treatment period (days 1), completion of treatment visit (day 2), and a follow-up visit (day $14 \pm 3$ days after last TAK063 dose). After each treatment period, subjects stayed overnight at the study site for observation and were discharged on day 2. Each participant completed the study when they completed the follow-up visit after their last treatment2.

Subjects checked in on day -1 of each treatment period during which vital signs, electrocardiogram (ECG), and laboratory safety tests were repeated. On day 1 of each treatment period, subjects received a 3-, $10-$, or $30-\mathrm{mg}$ TAK-063 dose or placebo tablets. Participants then relaxed, slept, or watched movies while remaining in the research environment. Ketamine $(0.12 \mathrm{mg} / \mathrm{kg}$ intravenous bolus over $60 \mathrm{~s}$ followed by a continuous infusion of $0.31 \mathrm{mg} / \mathrm{kg} / \mathrm{h}$ over approximately $150 \mathrm{~min}$ ) was administered $4 \mathrm{~h}$ after TAK-063 or placebo (Fig. 1). This ketamine dose was based on a previous study that achieved steady-state concentrations of $75 \mathrm{ng} / \mathrm{mL}$ and provided a robust BOLD signal with minimal psychotomimetic symptoms (De Simoni et al. 2013).

Pharmacodynamic (PD) assessments, such as the neuroimaging battery and memory tasks, were performed before and during ketamine infusion. Safety was continuously assessed during ketamine administration.

\section{Neuroimaging battery tests}

The scanning protocol for the current study was very comprehensive and included multiple MR approaches. This publication reports the BOLD fMRI data for the working memory task, but also resting state fMRI. The magnetic scanning portion of the neuroimaging test battery was initiated $20 \mathrm{~min}$ before ketamine dosing. Imaging was performed using a 3 Tesla Siemens Trio scanner. The MR scanning protocol was initiated 20 min before ketamine dosing; prior to scanning, a structural magnetization prepared rapid gradient echo (MPRAGE) scan was collected. As part of the scanning protocol, each subject received $20 \mathrm{~min}$ of scanning $-10 \mathrm{~min}$ arterial spin labeling (ASL) plus 10 min resting BOLD prior to ketamine infusion. After ketamine infusion, the resting state fMRI BOLD acquisition continued for an additional $10 \mathrm{~min}$. Next, a Spatial Working Memory task-induced fMRI BOLD acquisition was performed for $15 \mathrm{~min}$. Following these BOLD acquisitions, another 10 min ASL scan was acquired and a 10min T1 relaxometry scan was conducted. The MR scanning sequence ended with a 5-min MPRAGE acquisition.

\section{Image acquisition}

Fifty sequential gradient-echo echoplanar images (EPIs) sensitive to blood oxygenation level-dependent (BOLD) signal were collected in contiguous slices of $6-\mathrm{mm}$ thickness, with 3000-ms repetition time, 40-ms echo time, $20 \times 20$-cm field of view, a $64 \times 64$ image matrix, $70^{\circ}$ flip angle, and an in-plane resolution of $3 \times 3 \mathrm{~mm}$.

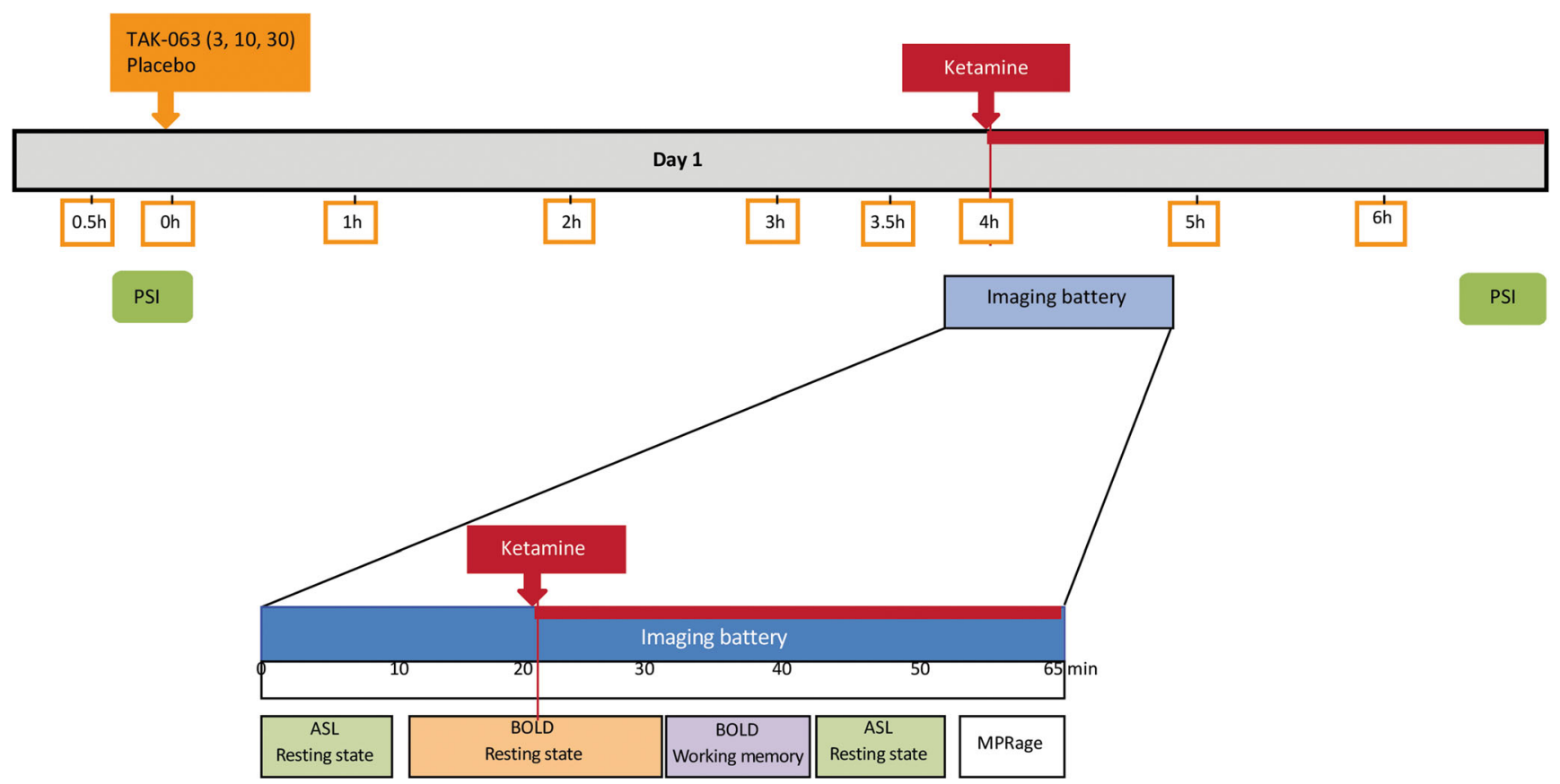

Fig. 1 Study design. ASL, arterial spin labeling; BOLD, blood oxygen level-dependent; MPRage, magnetization-prepared rapid acquisition with gradient echo 
A working memory challenge based on the expectancy A-X Continuous Performance Test (AX-CPT) paradigm was presented. The AX-CPT task was implemented in Eprime and presents 144 trials in four blocks of 36 trials. Before scanning, participants practiced the task (Barch et al. 2001; MacDonald et al. 2003).

\section{Image pre-processing}

We conducted a number of pre-processing steps to standardize examination of data after acquisition. These steps included (a) using the anatomical image (T1 weighted MPRAGE acquired for each subject at the beginning of the imaging session) to determine the parameters for spatial normalization to the EPI template image supplied with statistical parametric mapping (SPM) using a 12-parameter transformation; (b) registering functional images to correct for subject movement during the experimental paradigm; (c) normalizing functional images to transform images from analyzed subjects into the same standard space; (d) smoothing images (8-mm full width at half maximum [FWHM]) to minimize normalization artifacts and improve signal to noise ratio; (e) applying a high pass filter of $1200 \mathrm{~s}$ to the data following spatial smoothing to minimize the influence of very low frequency noise present in fMRI BOLD data without distorting the ketamine response profile.

\section{Image analyses}

The functional imaging analyses for the working memory task was performed using SPM8 (Wellcome Department of Cognitive Neurology, London, UK). Functional images were realigned to correct for motion-related variance components normalized to the standard Montreal Neurological Institute (MNI) EPI template (Talairach and Tournoux 1988; Friston et al. 1995a) and spatially smoothed with an 8-mm FWHM isotropic Gaussian kernel to allow for anatomical variation among subjects. The statistical parametric maps were generated using the general linear model in SPM8 (Friston et al. 1995b). Low-frequency noise was removed with a high-pass filter with a cutoff of $127 \mathrm{~s}$ applied to the fMRI time series at each voxel. Data from each subject for each task were analyzed with a fixed box-car function convolved with a model hemodynamic response function. Signal change was acquired from individual ROIs; a total of 22 ROIs were used in the study, including amygdala (left and right), anterior cingulate gyrus (left and right), striatum (caudate and putamen; left and right), dorsolateral prefrontal cortex (BA8 + BA9 + BA46; left and right), posterior cingulate gyrus (left and right), substantia nigra (left and right), ventrolateral prefrontal cortex (BA44 + BA45BA47; left and right), subgenual anterior cingulate cortex (BA25; left and right), thalamus (left and right), paracingulate gyrus (BA32; left and right), and hippocampus (left and right).

\section{Clinical and behavioral measures}

We collected a number of behavioral and side effect measures, including the Psychotomimetic States Inventory (PSI), the Columbia Suicide Severity Rating Scale (C-SSRS), and the CNS Vital Signs Cognitive Battery. These were exploratory measures and are not included in this report.

\section{Study endpoints}

The primary endpoint was ketamine-induced brain activity during the resting state in select regions of the brain including the anterior cingulate cortex, striatum, and ventrolateral prefrontal cortex. Secondary endpoints included pharmacokinetic (PK) parameters of TAK-063 (maximum plasma drug concentration $\left[C_{\max }\right]$, average steady-state plasma drug concentration $\left[C_{\mathrm{av}}\right]$, time to reach maximum plasma concentration $\left[t_{\mathrm{max}}\right]$, area under the plasma concentration-time curve for $24 \mathrm{~h}$ $\left[\mathrm{AUC}_{24}\right]$ ), and percentage of subjects experiencing at least one treatment-emergent adverse event (AE).

\section{Pharmacokinetic assessments}

Blood samples were collected for the determination of TAK063 and ketamine concentrations, and ketamine concentrations were used to verify intravenous delivery of ketamine. Blood samples for determination of TAK-063 concentration were collected at pre-dose and at $0.5,2,3,4.5,6,8,10,12$, and $24 \mathrm{~h}$ post dose. Blood samples for determination of ketamine concentration were collected at $0.5,1,2$, and $2.5 \mathrm{~h}$ after administration of ketamine. The target ketamine concentration was established at $75 \mathrm{ng} / \mathrm{mL}$. PK samples were not collected from the arm in which ketamine was administered.

\section{Safety assessments}

AEs, clinical laboratory results, ECG, vital signs, and suicide assessments were recorded after administration of TAK-063 and throughout the remainder of the study. Intensity of AEs was classified as mild, moderate, and severe, and each AE was assessed for association with study drug. AEs were assessed throughout the 2-day study visit, at the three treatment visits, at the study exit, and at the follow-up visit. ECG, vital signs, and clinical laboratory results were assessed at visit 1 during screening, day -1 , day 1 of each treatment period, day 2 of period 3 (study exit), the early termination visit, and the follow-up visit.

\section{Statistical analyses}

In this study, the safety set, used for demographic and safety evaluations, included subjects who receive at least one dose of study medication. The PK set included all subjects in the 
safety set with at least one available drug concentration measurement. The PD set included all subjects in the safety set and with at least one valid PD assessment.

This study was not powered for any comparison, and the number of subjects was considered to be suitable for fulfilling study objectives. All statistical tests were twotailed at $\alpha=0.05$ level of significance. For the analysis of the neuroimaging battery test results, effect sizes of $>$ 0.3 were considered potentially meaningful, even without power to detect statistical significance. PK parameters of TAK-063 were generated using WinNonlin Version 6.3. fMRI images were analyzed using SPM8 and Matlab. Percent signal change for resting state data was calculated between pre-ketamine and post-ketamine infusion based on a priori ROIs using SPM's Anatomy Toolbox (Yurgelun-Todd et al. 2016). Percent signal change for the working memory task was calculated for the task versus baseline in the post-ketamine condition using the same ROI used for resting state (Yurgelun-Todd et al. 2016). An analysis of variance (ANOVA) model was used to perform the analyses for BOLD signal changes (Yurgelun-Todd et al. 2016). Cohen's $d$ effect sizes were calculated as

$d=\frac{\widehat{\mu_{t}}-\widehat{\mu_{p}}}{s_{p}}$

where $d$ is the effect size and $\widehat{\mu_{t}}$ and $\widehat{\mu_{p}}$ are the leastsquare means for a TAK-063 dose and placebo regimen, respectively, from the ANOVA model. $s_{p}$ is the pooled standard deviation from both regimens. No adjustments were made for multiple comparisons.

\section{Results}

\section{Demographics}

In total, 27 subjects were enrolled and randomly assigned to treatment schedules as follows: 22 to regimen A (placebo + ketamine), 14 to regimen B (3 mg TAK-063 + ketamine), 15 to regimen $\mathrm{C}$ (30 mg TAK063 + ketamine), and 14 to regimen D (10 mg TAK063 + ketamine); two subjects received $300 \mathrm{mg}$ of TAK-063 and were not included in the analysis. A total of 20 subjects completed the study (treatment sequences are shown in Online Resource Supplementary Fig S1). All subjects were male, and most were white (88.9\%) and non-Hispanic or non-Latino (96.3\%) (Online Resource Supplementary Table S2). Mean age, height, weight, and BMI were generally similar across dosing groups (Online Resource Supplementary Table S2).

\section{Pharmacokinetics}

After oral administration, the rate of TAK-063 absorption appeared to be moderate, with a median $t_{\max }$ value of $3 \mathrm{~h}$ (range $0.5-6 \mathrm{~h}$ ). Dose proportionality analysis for $C_{\max }$ and AUC of TAK-063 showed a close to dose proportional increase in exposure across the dose range (3-30 mg). After $C_{\max }$ was reached, plasma concentrations declined in a biphasic manner, with an initial rapid phase followed by a slower terminal phase. $\mathrm{AUC}_{24}$ values increased with TAK-063 dose $(3 \mathrm{mg}$ : $111.3 \mathrm{ng} \cdot \mathrm{h} / \mathrm{mL} ; 10 \mathrm{mg}: 549.2 \mathrm{ng} \cdot \mathrm{h} / \mathrm{mL} ; 30 \mathrm{mg}: 1296.3 \mathrm{ng} \cdot \mathrm{h} /$ $\mathrm{mL})$. Average concentration $\left(C_{\mathrm{av}}\right)$ values for 3,10 , and $30 \mathrm{mg}$ doses of TAK-063 were $4.63,22.9$, and $53.9 \mathrm{ng} / \mathrm{mL}$, respectively. Overall, the PK profile of TAK-063 was similar to that described in the single-rising dose study, with some differences in TAK-063 M-I PK (Tsai et al. 2016).

Following ketamine administration, mean ketamine concentrations ranged from 69.9 to $83.3 \mathrm{ng} / \mathrm{mL}$ at $30 \mathrm{~min}$ post dose, irrespective of co-administration of placebo or TAK-063 from 3 to $30 \mathrm{mg}$. During continuous infusion, plasma concentrations of ketamine reached mean $C_{\max }$ values ranging from 167.7 to $178.7 \mathrm{ng} / \mathrm{mL}$. $t_{\max }$ values for ketamine ranged from 2.3 to $2.5 \mathrm{~h}$, and overall $C_{\mathrm{av}}$ values ranged from 118.6 to 124.6 $\mathrm{ng} / \mathrm{mL}$. Inter-subject variability of $C_{\max }$ and $C_{\mathrm{av}}$ was generally moderate across doses.

\section{fMRI BOLD signal}

Ketamine administration increased fMRI BOLD resting signal in the placebo group in most regions studied. A decrease in fMRI BOLD was observed in cingulate/BA25. These results are consistent with previous reports (De Simoni et al. 2013).

TAK-063 exerted region-specific effects on fMRI BOLD signal during the resting state before ketamine infusion (Fig. 2). Compared with placebo, TAK-063 increased Cohen's effect size for resting fMRI BOLD signal across all dose groups at the left and right posterior cingulate cortex and right thalamus. TAK-063 reduced Cohen's effect size for resting fMRI BOLD signal in all dose groups at the anterior cingulate cortex, striatum, ventrolateral prefrontal cortex, and dorsolateral prefrontal cortex. Following ketamine administration, TAK-063 had a meaningful effect attenuating ketamineinduced changes on resting state fMRI BOLD signals in many key regions examined (Fig. 3). Even though the study was not powered to demonstrate statistical significance, there were noticeable regional fluctuations (Fig. 4), and the following regions showed an absolute effect size greater than 0.3 (threshold established for clinical meaningfulness) and a statistically significant difference $(P<0.05)$ vs placebo: left striatum (3 mg TAK-063; $P=0.039 ; 95 \%$ confidence interval $[\mathrm{CI}]-0.5955,-0.0162$ ), right striatum (3-mg TAK-063; $P=$ $0.020 ; 95 \% \mathrm{CI}-0.5985,-0.0554)$, left substantia nigra (30mg TAK-063; $P=0.012 ; 95 \% \mathrm{CI}-0.7724,-0.1031)$, and 

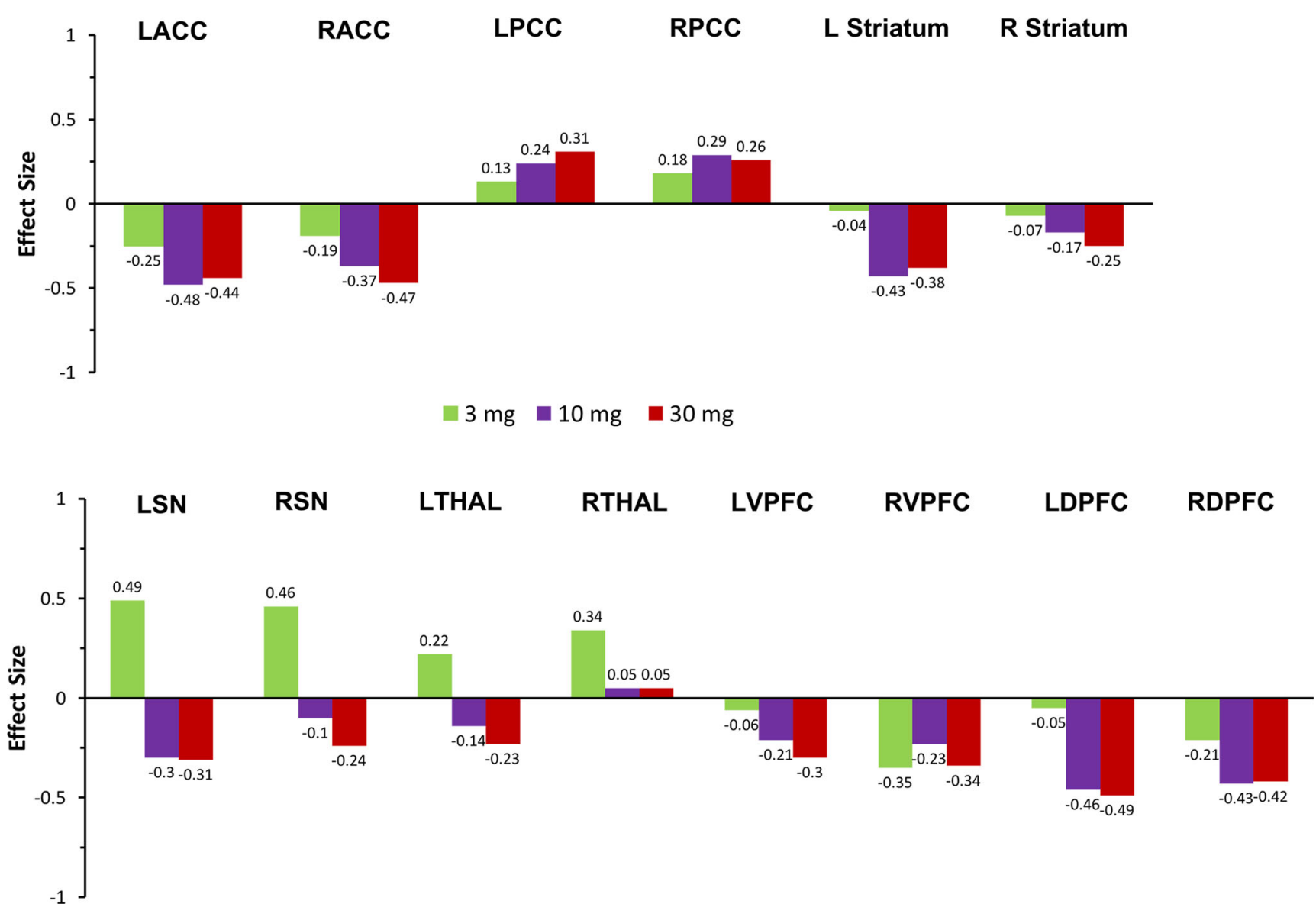

Fig. 2 Effects of TAK-063 on resting fMRI BOLD signal before ketamine infusion. Effect sizes were calculated using least squares mean data compared with placebo from the ANOVA model. $f M R I$, functional magnetic resonance imaging; $B O L D$, blood oxygen level-dependent; $L A C C$, left anterior cingulate cortex; $L D P F C$, left dorsolateral prefrontal cortex; $L P C C$, left posterior cingulate cortex; $L S N$, left substantia nigra; $L$

right ventrolateral prefrontal cortex (30-mg TAK-063; $P=$ $0.031 ; 95 \%$ CI $-0.7912,-0.0415)$. However, the effect sizes at the 10-mg TAK-063 dose were generally smaller relative to the effects of other dose groups during assessment of ketamine-induced BOLD changes in the resting state. The average ketamine-induced BOLD signal changes in the placebo regimen (regimen A) are shown in Fig. 5.

During the working memory task and across all doses, TAK-063 decreased the Cohen's effect size for fMRI BOLD signal in many brain regions (Fig. 6). The only exceptions were in the right substantia nigra, left thalamus, and left ventrolateral prefrontal cortex in the 10-mg TAK-063 group, where small non-negative effects (effect size $<0.15$ ) were evident, and the left amygdala, which showed a small non-negative effect in the $30-\mathrm{mg}$ TAK-063 group. The following regions showed an absolute effect size greater than 0.3 and statistically significant difference $(P<0.05)$ vs. placebo: right ventrolateral
Striatum, left striatum; $L T H A L$, left thalamus; $L V P F C$, left ventrolateral prefrontal cortex; $R A C C$, right anterior cingulate cortex; $R D P F C$, right dorsolateral prefrontal cortex; $R P C C$, right posterior cingulate cortex; $R S N$, right substantia nigra; $R$ Striatum, right striatum; $R T H A L$, right thalamus; $R V P F C$, right ventrolateral prefrontal cortex

prefrontal cortex $(3-\mathrm{mg} ; P=0.036 ; 95 \%$ CI $1.0251,-0.0369)$ and left dorsolateral prefrontal cortex $(30-\mathrm{mg} ; P=0.043 ; 95 \%$ CI -1.0107 , 0.0163). As observed during the resting state, effect sizes for the 10-mg TAK-063 group at many regions were generally smaller compared with other dose groups.

Ketamine increased activation in all ROIs examined (Fig. 7). The attenuation in ketamine-induced BOLD signal changes seen with TAK-063 pre-treatment (vs placebo pretreatment) demonstrated focal patterns of activation similar to what would be expected in healthy control subjects in the absence of ketamine administration (Online Resource Supplementary Fig S2).

Together, these results suggest that TAK-063 ameliorated ketamine-induced changes in fMRI BOLD signal during the resting state and working memory task. 


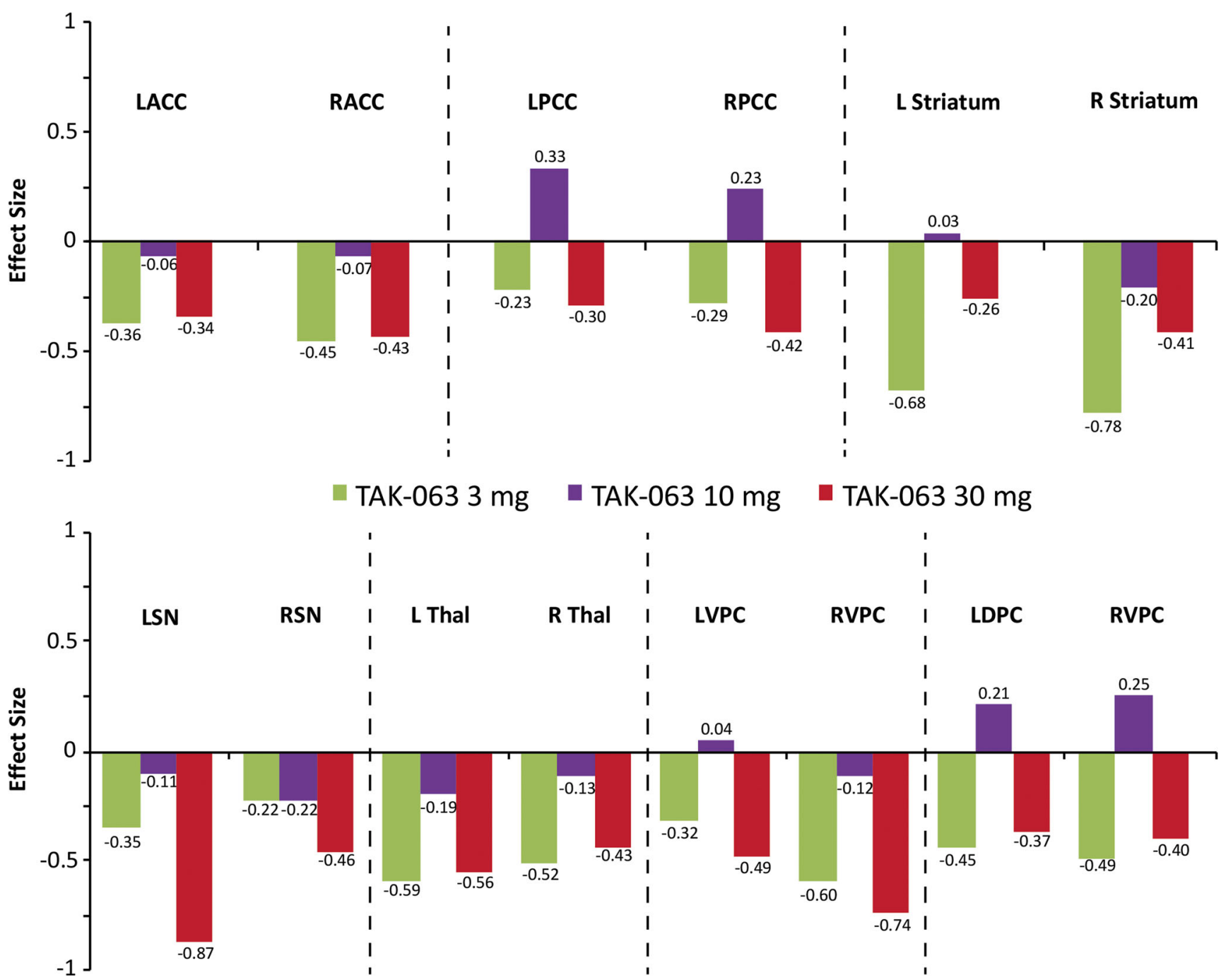

Fig. 3 Effects of TAK-063 treatment on ketamine-induced changes in fMRI BOLD signal during resting state. Effect sizes were calculated using least squares mean data as compared to placebo from the ANOVA model. $f M R I$, functional magnetic resonance imaging; $B O L D$, blood oxygen level-dependent; $L A C C$, left anterior cingulate cortex; $L D P C$, left dorsolateral prefrontal cortex; $L P C C$, left posterior cingulate

\section{Safety}

A total of 24 subjects (88.9\%) experienced AEs that were related to the study drug, and all were mild to moderate in severity. The most frequently reported AEs ( $\geq 2$ subjects) were vomiting (63.0\%), nausea (40.7\%), somnolence (40.7\%), and fatigue (25.9\%). No changes from baseline to post dose in vital signs (serum chemistry, hematology, and urinalysis), clinical laboratory tests, and 12-lead ECGs were considered clinically significant. No deaths or serious AEs occurred during the study, and AEs led one participant to discontinue study drug. These results are consistent with the single-rising dose study (Tsai et al. 2016).

Overall, AEs consistent with extrapyramidal symptoms (EPS) were experienced by two subjects in the TAK-063 cortex; $L S N$, left substantia nigra; $L$ Striatum, left striatum; $L$ Thal, left thalamus; $L V P C$, left ventrolateral prefrontal cortex; $R A C C$, right anterior cingulate cortex; $R P C C$, right posterior cingulate cortex; $R S N$, right substantia nigra; $R$ Striatum, right striatum; $R$ Thal, right thalamus; $R V P C$, right ventrolateral prefrontal cortex

group (8.0\%), and no subjects in the placebo group experienced symptoms of EPS. One subject in the 30-mg TAK-063 group experienced restlessness and another in the $300-\mathrm{mg}$ TAK-063 group experienced muscle tightness and restlessness. One subject receiving $300 \mathrm{mg}$ of TAK-063 discontinued because of EPS. Together, these results suggest that TAK-063 was safe and well tolerated during the study.

\section{Discussion}

The modulation of the BOLD signal by TAK-063 may be related to the augmentation of the direct and indirect striatal output. TAK-063 attenuated ketamine-induced changes in BOLD signal in multiple regions of the brain during the 
Fig. 4 Regional effects of different doses of TAK-063 treatment on ketamine-induced changes in $\mathrm{AMRI}$ BOLD signal during resting state. $f M R I$, functional magnetic resonance imaging; $B O L D$, blood oxygen leveldependent
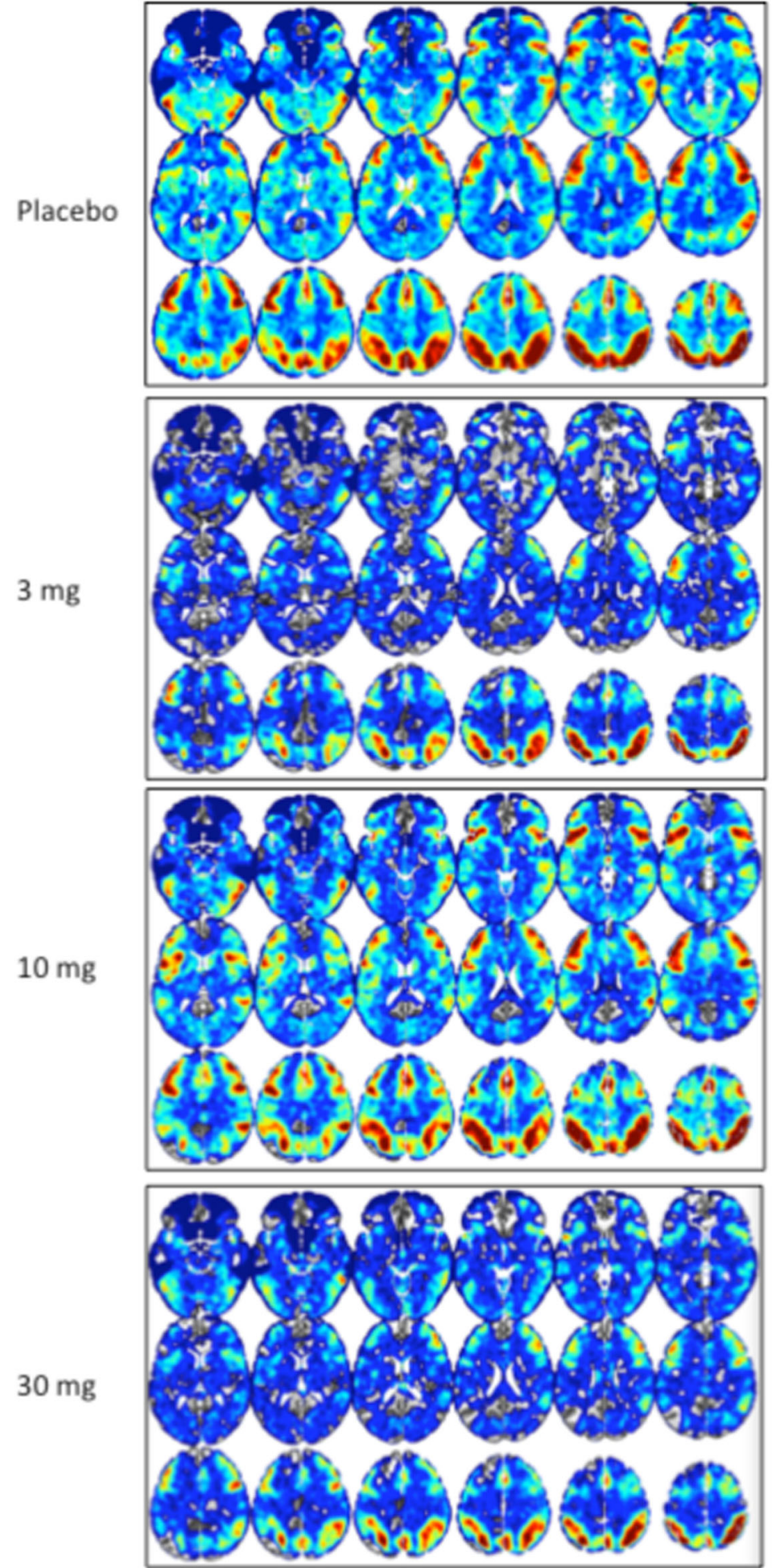

resting state and working memory tasks. This trend was similar to a previous study in which risperidone reduced ketamine-induced BOLD signal in multiple regions of the brain (Doyle et al. 2013). However, the BOLD resting state results from our study do not align with a previous ASL-based study of ketamine-induced changes in brain perfusion in which risperidone increased ketamine-induced perfusion changes (Shcherbinin et al. 2015). Taken together, these results suggest that the attenuation of BOLD changes seen with risperidone, and possibly with TAK-063, is not due to generalized changes in blood flow. The PK of TAK-063 were consistent with those of previous reports (Tsai et al. 2016), and 


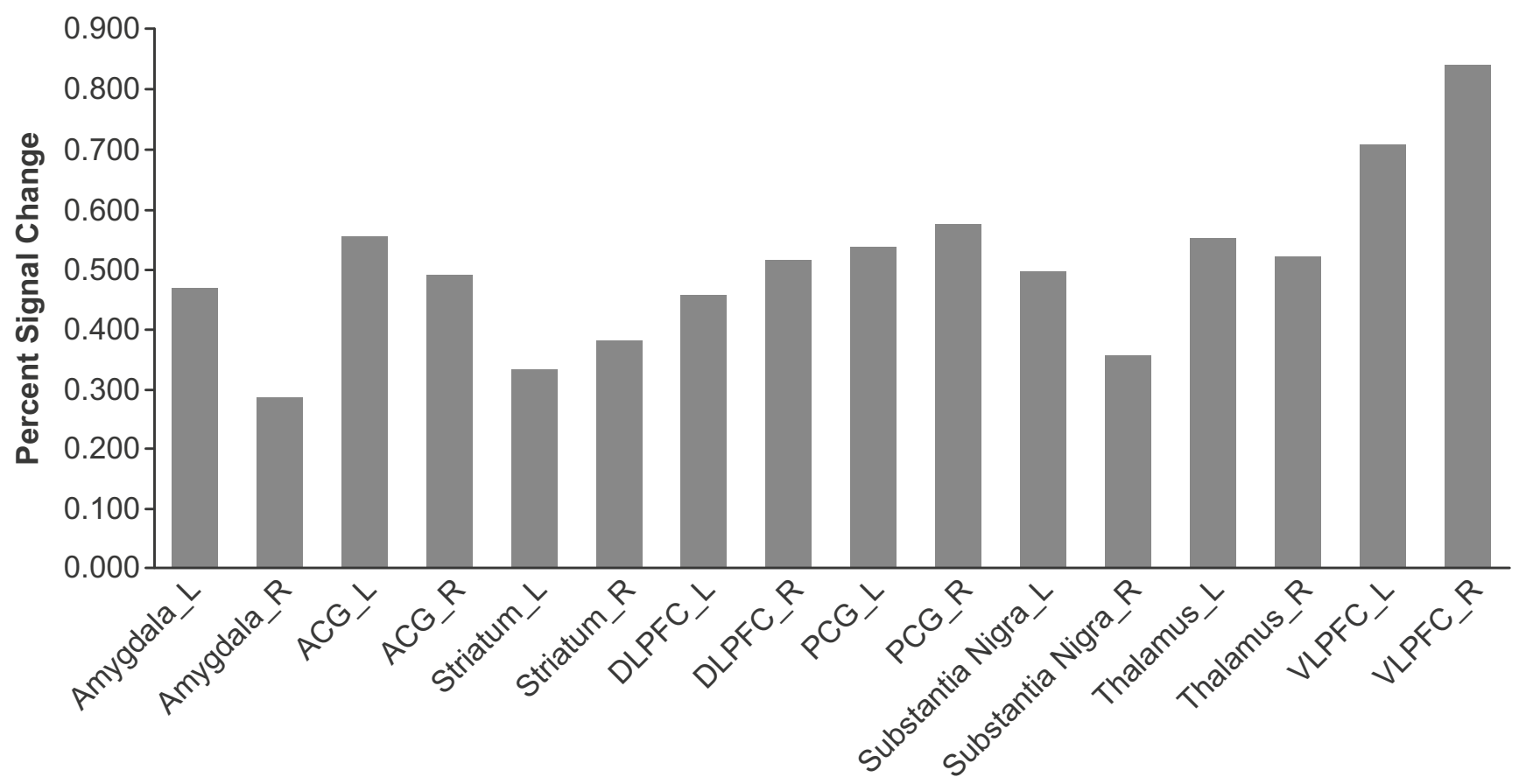

Fig. 5 Average ketamine-induced signal change for $\mathrm{AMRI} B \mathrm{BOL}$ resting state data in the placebo regimen (regimen A). $f M R I$, functional magnetic resonance imaging; $B O L D$, blood oxygen level-dependent; $A C G$, anterior cingulate gyrus; $D L P F C$, dorsolateral prefrontal cortex; $L$, left; $P C G$, posterior cingulate cortex; $R$, right; $V L P F C$, ventrolateral prefrontal cortex
TAK-063 plasma concentrations exhibited biphasic behavior, as has been observed in previous single- and multiple-rising dose studies (Goldsmith et al. 2017; Tsai et al. 2016).

While the study was not powered for any comparison, there was a consistent attenuation of the ketamineinduced signal increases in BOLD signal with TAK-063 administration that is generally consistent with the effects of risperidone on ketamine-induced increases in BOLD (Doyle et al. 2013). Although not definitively known, the attenuation of ketamine-induced increases in BOLD in this translational model of schizophrenia may be suggestive of potential antipsychotic effects of TAK-063. The effects on ketamine-induced changes in BOLD signal were most consistent in the 3- and 30-mg dose groups, especially during the working memory task. Particularly in cortical regions, the effects were generally dose-dependent in the 3- and 30-mg dose groups. In general, the magnitude of change in the 10mg group was less, although the 10-mg TAK-063 dose results were more consistent in the cortical regions of the brain; the reasons for these differences are not known.

TAK-063 was well tolerated at all doses of the study. No serious AEs were experienced by subjects receiving TAK063, and most AEs were of mild to moderate severity. Only two subjects experienced symptoms of EPS, and one of the two subjects received a relatively high TAK-063 dose $(300 \mathrm{mg})$ that was later changed to $10 \mathrm{mg}$ (according to protocol amendment 3 ). The safety profile of TAK-063 closely resembled that of the single-rising dose study in which only one subject experienced EPS-like symptoms (Tsai et al. 2016).

PDE-10 is selectively expressed on the medium spiny neurons of the striatum. This enzyme inhibits the dephosphorylation of G-protein receptors, which are coupled to the inhibition of cAMP and cGMP, and therefore act in general like D2 dopamine antagonists (Nishi et al. 2008; Sano et al. 2008; Xie et al. 2006; Gresack et al. 2014). To date, PDE10A inhibitors have not shown clinical efficacy in the treatment of schizophrenia, and the predictability of this translational model of schizophrenia has not been definitively established. TAK-063 is a potent, highly selective, and orally active PDE10A inhibitor that produces a balanced activation of downstream cAMP and CGMP signals in both direct and indirect pathways medium spiny neurons - balanced activation of these pathways may be critical for an antipsychotic-like effect (Suzuki and Kimura 2018; Suzuki et al. 2016). In preclinical models, TAK-063 has shown effects that are consistent with potential antipsychotic efficacy, and has also attenuated the increases in BOLD in anesthetized rats; additionally, in clinical studies of individuals with schizophrenia, potential antipsychotic-like effects were observed. In a 6-week phase 2 

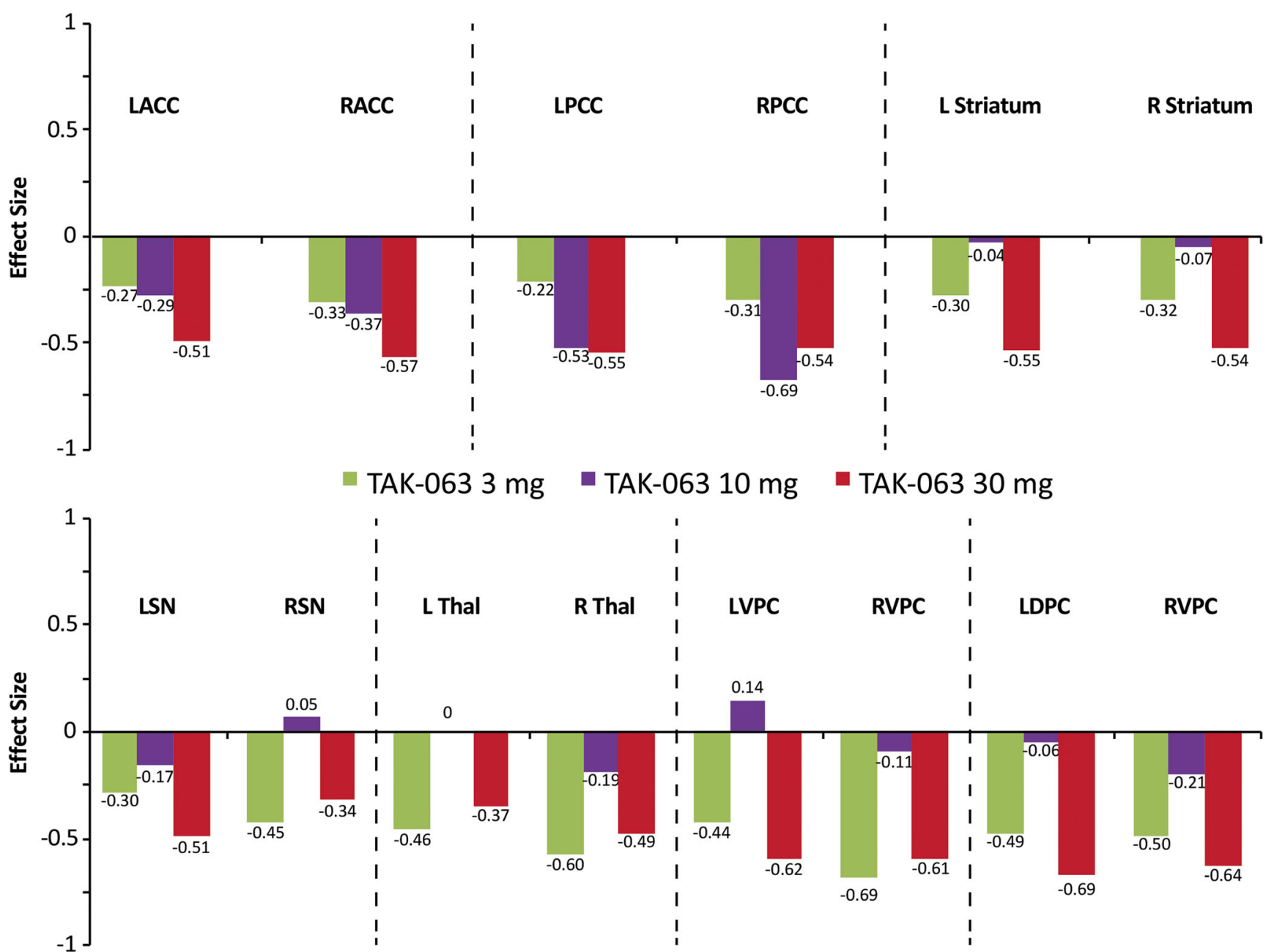

Fig. 6 Effects of TAK-063 treatment on ketamine-induced changes in FMRI BOLD signal during the execution of working memory tasks. Effect sizes were calculated using least squares mean data compared with placebo from the ANOVA model. $f M R I$, functional magnetic resonance imaging; $B O L D$, blood oxygen level-dependent; $L A C C$, left anterior cingulate cortex; $L D P C$, left dorsolateral prefrontal cortex; $L P C C$, left posterior cingulate cortex; $L S N$, left substantia nigra; $L$ Striatum, left striatum; $L$ Thal, left thalamus; $L V P C$, left ventrolateral prefrontal cortex; $R A C C$, right anterior cingulate cortex; $R P C C$, right posterior cingulate cortex; $R S N$, right substantia nigra; $R$ Striatum, right striatum; $R$ Thal, right thalamus; $R V P C$, right ventrolateral prefrontal cortex study, the difference between the 20-mg dose of TAK-063 and placebo did not meet its primary endpoint (change from baseline in Positive and Negative Syndrome Scale [PANSS] score at week 6). However, statistically significant effects were observed for several secondary endpoints, and the overall results were suggestive of antipsychotic efficacy. Unfortunately, the interpretation of this study was confounded by a relatively large change from baseline in PANSS score in the placebo group, the lack of dose-ranging comparisons, and an active reference (Macek et al. 2017). Further clinical studies are required to assess the potential antipsychotic effects of TAK-063.

Limitations of this study include the small sample size and the significant dropout rate (20 out of 27 subjects completed the study). All subjects were male (homogeneous sample), which may not allow to generalize findings to females. The exposure to ketamine was longer than that used in other studies ( $4 \mathrm{~h}$ as opposed to $40 \mathrm{~min}$ ), making comparisons across studies difficult. Given the observed side effects with the 300mg dose of TAK-063, we were unable to complete the doseresponse curve that was initially planned $(3-300 \mathrm{mg}$ ). Additionally, no adjustments for multiplicity were made for statistical analyses.

Additional analyses are required to examine the possible contributions of changes in blood flow to the observations. ASL was measured in this study, and further analyses of these data are the next logical step for assessing the impact of the findings. These results may help to further understand the PD effects of TAK-063 and other PDE10A inhibitors. 


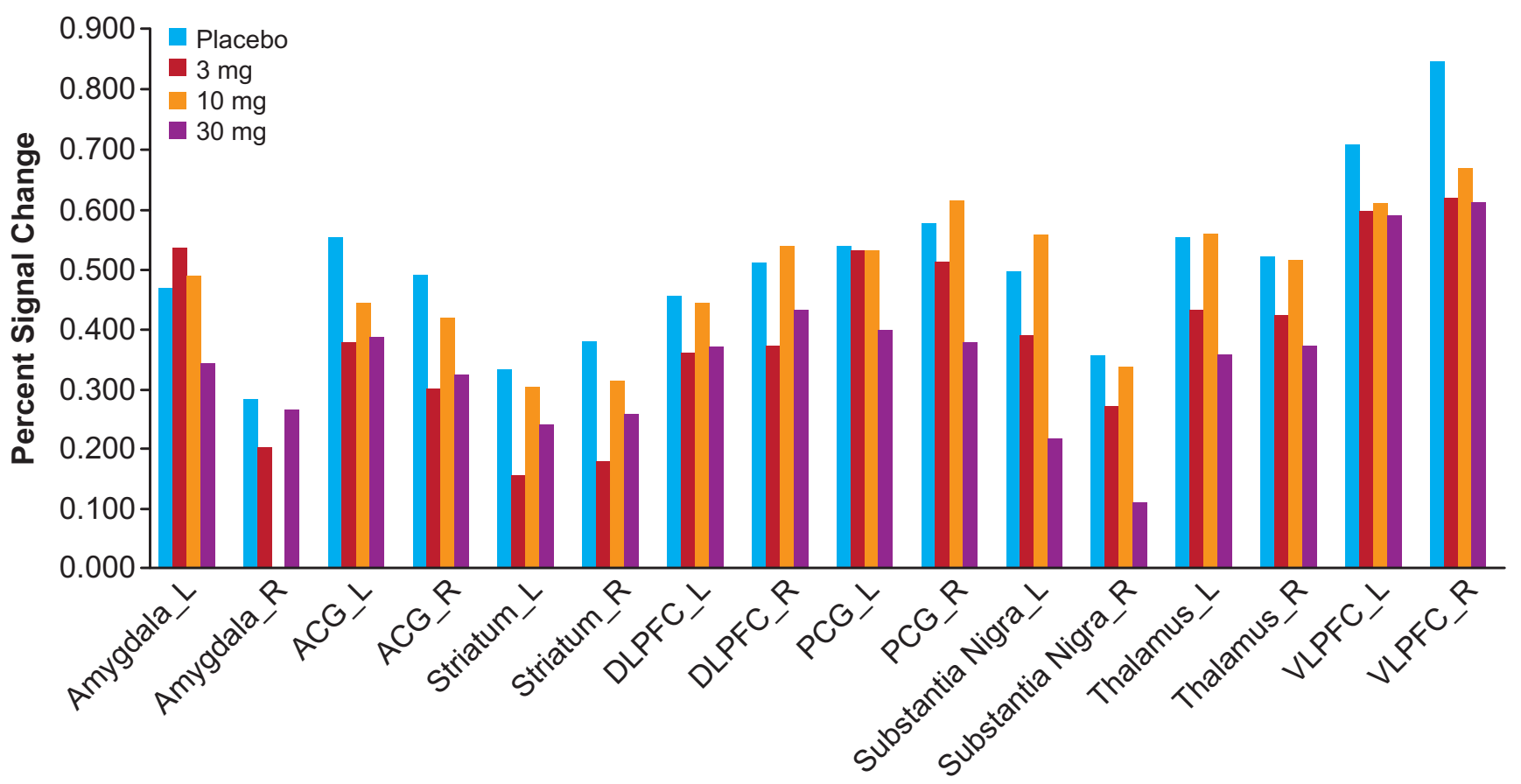

Fig. 7 Percent ketamine-induced BOLD signal change for resting state during placebo and TAK-063 regimens in evaluated ROIs. BOLD, blood oxygen level-dependent; ROIs, regions of interest; $A C G$, anterior cingulate gyrus; $D L P F C$, dorsolateral prefrontal cortex; $L$, left; $P C G$, posterior cingulate cortex; $R$, right; $V L P F C$, ventrolateral prefrontal cortex
Acknowledgments Some data included in this manuscript were presented in abstract form (Yurgelun-Todd D, Renshaw P, Goldsmith P, Xie J, Uz T, Macek T. The PDE-10A Inhibitor TAK-063 Reverses KetamineInduced Changes in fMRI BOLD Signal. Society of Biological Psychiatry Meeting 2016, and Yurgelun-Todd D, Renshaw P, Goldsmith P, Xie J, Uz T, Macek T. The PDE-10A Inhibitor TAK-063 Reverses Ketamine-Induced Changes in fMRI BOLD Signal. Schizophrenia International Research Society Conference 2016). We thank Jinhui Xie for her contribution to this study.

Author contributions Deborah Yurgelun-Todd and Perry Renshaw assisted with development of the imaging protocol, carried out the study, analyzed imaging data, and assisted with data interpretation. Thomas A. Macek, Tolga Uz, and Paul Goldsmith assisted with the development of the study protocol and provided sponsor oversight of the execution and summarization of the study.

Funding information The clinical study was funded by Takeda Development Center Americas, Inc. Medical writing assistance was provided by Stephanie Agbu, PhD, and Jake Edelstein, $\mathrm{PhD}$, of inVentiv Medical Communications, LLC, a Syneos Health $^{\mathrm{TM}}$ group company, and supported by Takeda Development Center Americas, Inc.

\section{Compliance with ethical standards}

Conflict of interest Thomas A. Macek and Tolga Uz were employees of Takeda Development Center Americas, Inc., Deerfield, IL, at the time of this study, and Paul Goldsmith was an employee of Takeda Development Centre Europe Ltd., London, UK, at the time of this study. Deborah Yurgelun-Todd and Perry Renshaw have no conflicts of interest to disclose.
Open Access This article is distributed under the terms of the Creative Commons Attribution 4.0 International License (http:// creativecommons.org/licenses/by/4.0/), which permits unrestricted use, distribution, and reproduction in any medium, provided you give appropriate credit to the original author(s) and the source, provide a link to the Creative Commons license, and indicate if changes were made.

\section{References}

Abi-Saab WM, D'Souza DC, Moghaddam B, Krystal JH (1998) The NMDA antagonist model for schizophrenia: promise and pitfalls. Pharmacopsychiatry 31(Suppl 2):104-109. https://doi.org/10.1055/ s-2007-979354

Barch DM, Carter CS, Braver TS, Sabb FW, MacDonald A 3rd, Noll DC, Cohen JD (2001) Selective deficits in prefrontal cortex function in medication-naive patients with schizophrenia. Arch Gen Psychiatry 58(3):280-288

Citrome L (2014) Unmet needs in the treatment of schizophrenia: new targets to help different symptom domains. J Clin Psychiatry 75(Suppl 1):21-26. https://doi.org/10.4088/JCP.13049su1c.04

De Simoni S, Schwarz AJ, O'Daly OG, Marquand AF, Brittain C, Gonzales C, Stephenson S, Williams SC, Mehta MA (2013) Testretest reliability of the BOLD pharmacological MRI response to ketamine in healthy volunteers. Neuroimage 64:75-90. https://doi. org/10.1016/j.neuroimage.2012.09.037

Doyle OM, De Simoni S, Schwarz AJ, Brittain C, O'Daly OG, Williams SC, Mehta MA (2013) Quantifying the attenuation of the ketamine pharmacological magnetic resonance imaging response in humans: a validation using antipsychotic and glutamatergic agents. J 
Pharmacol Exp Ther 345:151-160. https://doi.org/10.1124/jpet.112. 201665

Driesen NR, McCarthy G, Bhagwagar Z, Bloch MH, Calhoun VD, D'Souza DC, Gueorguieva R, He G, Leung HC, Ramani R, Anticevic A, Suckow RF, Morgan PT, Krystal JH (2013) The impact of NMDA receptor blockade on human working memory-related prefrontal function and connectivity. Neuropsychopharmacology 38:2613-2622. https://doi.org/10.1038/npp.2013.170

Friston KJ, Ashburner J, Frith CD, Poline JB, Heather JD, Frackowiak RSJ (1995a) Spatial registration and normalization of images. Hum Brain Mapp 2:165-189

Friston KJ, Frith CD, Frackowiak RS, Turner R (1995b) Characterizing dynamic brain responses with fMRI: a multivariate approach. Neuroimage 2(2):166-172

Frohlich J, Van Horn JD (2014) Reviewing the ketamine model for schizophrenia. J Psychopharmacol 28:287-302. https://doi.org/10. 1177/0269881113512909

Goldsmith P, Affinito J, McCue M, Tsai M, Roepcke S, Xie J, Gertsik L, Macek TA (2017) A randomized multiple dose pharmacokinetic study of a novel pde10A inhibitor TAK-063 in subjects with stable schizophrenia and Japanese subjects and modeling of exposure relationships to adverse events. Drugs R D 17:631-643. https://doi. org/10.1007/s40268-017-0214-8

Gresack JE, Seymour PA, Schmidt CJ, Risbrough VB (2014) Inhibition of phosphodiesterase 10A has differential effects on dopamine D1 and D2 receptor modulation of sensorimotor gating. Psychopharmacology 231(10):2189-2197

Lahti AC, Weiler MA, Tamara Michaelidis BA, Parwani A, Tamminga CA (2001) Effects of ketamine in normal and schizophrenic volunteers. Neuropsychopharmacology 25:455-467. https://doi.org/10. 1016/S0893-133X(01)00243-3

Lofwall MR, Griffiths RR, Mintzer MZ (2006) Cognitive and subjective acute dose effects of intramuscular ketamine in healthy adults. Exp Clin Psychopharmacol 14:439-449. https://doi.org/10.1037/10641297.14.4.439

MacDonald AW 3rd, Pogue-Geile MF, Johnson MK, Carter CS (2003) A specific deficit in context processing in the unaffected siblings of patients with schizophrenia. Arch Gen Psychiatry 60(1):57-65

Macek TA, McCue M, Xie J, Johnstone J, Boeijinga P (2016a) Abstract T155 TAK-063 increases gamma synchrony in subjects with schizophrenia. NPJ Schizophr 2:58

Macek TA, McCue M, Xie J, Wesnes K (2016b) Abstract T154 The effects of TAK-063 on cognition in a multiple dose, phase 1 study in healthy Japanese volunteers and subjects with schizophrenia are consistent with its somnolent effects. NPJ Schizophr 2:57

Macek T, McCue M, Ogrinc F, Hanson E, Goldsmith P, Affinito J, Mahableshwarkar AR (2017) M20. A phase 2, randomized, double-blind, placebo-controlled, parallel-group, 6-week study to evaluate the efficacy and safety of TAK-063 in subjects with an acute exacerbation of schizophrenia. Schizophr Bull 43:S218-S218

Millier A, Schmidt U, Angermeyer MC, Chauhan D, Murthy V, Toumi M, Cadi-Soussi N (2014) Humanistic burden in schizophrenia: a literature review. J Psychiatr Res 54:85-93. https://doi.org/10. 1016/j.jpsychires.2014.03.021

Morgan CJ, Mofeez A, Brandner B, Bromley L, Curran HV (2004) Acute effects of ketamine on memory systems and psychotic symptoms in healthy volunteers. Neuropsychopharmacology 29:208-218. https:// doi.org/10.1038/sj.npp.1300342

Nishi A, Kuroiwa M, Miller DB, O'Callaghan JP, Bateup HS, Shuto T, Sotogaku N, Fukuda T, Heintz N, Greengard P, Snyder GL (2008) Distinct roles of PDE4 and PDE10A in the regulation of cAMP/ PKA signaling in the striatum. J Neurosci 28(42):10460-10471
Sano H, Nagai Y, Miyakawa T, Shigemoto R, Yokoi M (2008) Increased social interaction in mice deficient of the striatal medium spiny neuron-specific phosphodiesterase. 10A2. J Neurochem 105(2): 546-556

Seeger TF, Bartlett B, Coskran TM, Culp JS, James LC, Krull DL, Lanfear J, Ryan AM, Schmidt CJ, Strick CA, Varghese AH, Williams RD, Wylie PG, Menniti FS (2003) Immunohistochemical localization of PDE10A in the rat brain. Brain Res 985:113-126

Shcherbinin S, Doyle O, Zelaya FO, de Simoni S, Mehta MA, Schwarz AJ (2015) Modulatory effects of ketamine, risperidone and lamotrigine on resting brain perfusion in healthy human subjects. Psychopharmacology 232:4191-4204. https://doi.org/10.1007/ s00213-015-4021-z

Shiraishi E, Suzuki K, Harada A, Suzuki N, Kimura H (2016) the phosphodiesterase 10A selective inhibitor TAK-063 improves cognitive functions associated with schizophrenia in rodent models. J Pharmacol Exp Ther 356:587-595. https://doi.org/10.1124/jpet. 115.230482

Suzuki K, Kimura H (2018) TAK-063, a novel PDE10A inhibitor with balanced activation of direct and indirect pathways, provides a unique opportunity for the treatment of schizophrenia. CNS Neurosci Ther 24(7):604-614. https://doi.org/10.1111/cns.12798

Suzuki K, Harada A, Shiraishi E, Kimura H (2015) In vivo pharmacological characterization of TAK-063, a potent and selective phosphodiesterase $10 \mathrm{~A}$ inhibitor with antipsychotic-like activity in rodents. J Pharmacol Exp Ther 352:471-479. https://doi.org/10.1124/ jpet.114.218552

Suzuki K, Harada A, Suzuki H, Miyamoto M, Kimura H (2016) TAK063, a PDE10A inhibitor with balanced activation of direct and indirect pathways, provides potent antipsychotic-like effects in multiple paradigms. Neuropsychopharmacology 41:2252-2262

Talairach J, Tournoux P (1988) Co-planar stereotaxic atlas of the human brain: 3-dimensional proportional system: an approach to cerebral imaging. Thieme Medical Publishers, Inc., New York

Tomimatsu Y, Cash D, Suzuki M, Suzuki K, Bernanos M, Simmons C, Williams SC, Kimura H (2016) TAK-063, a phosphodiesterase 10A inhibitor, modulates neuronal activity in various brain regions in phMRI and EEG studies with and without ketamine challenge. Neuroscience 339:180-190. https://doi.org/10.1016/j.neuroscience. 2016.10.006

Tsai M, Chrones L, Xie J, Gevorkyan H, Macek TA (2016) A phase 1 study of the safety, tolerability, pharmacokinetics, and pharmacodynamics of TAK-063, a selective PDE10A inhibitor. Psychopharmacology 233:3787-3795. https://doi.org/10.1007/ s00213-016-4412-9

Xie Z, Adamowicz WO, Eldred WD, Jakowski AB, Kleiman RJ, Morton DG, Stephenson DT, Strick CA, Williams RD, Menniti FS (2006) Cellular and subcellular localization of PDE10A, a striatumenriched phosphodiesterase. Neuroscience 139(2):597-607

Yamakura T, Chavez-Noriega LE, Harris RA (2000) Subunit-dependent inhibition of human neuronal nicotinic acetylcholine receptors and other ligand-gated ion channels by dissociative anesthetics ketamine and dizocilpine. Anesthesiology 92:1144-1153

Yurgelun-Todd D, Renshaw P, Goldsmith P, Xie J, Uz T, Macek TA (2016) Abstract T156 The PDE-10A inhibitor TAK-063 reverses ketamine-induced changes in fMRI BOLD signal. NPJ Schizophrenia 2:-58

Publisher's note Springer Nature remains neutral with regard to jurisdictional claims in published maps and institutional affiliations. 